\title{
Lightweight Spatial Conjunctive Query Answering Using Keywords ${ }^{\star}$
}

\author{
Thomas Eiter, Thomas Krennwallner, and Patrik Schneider \\ Institut für Informationssysteme, Technische Universität Wien \\ Favoritenstraße 9-11, A-1040 Vienna, Austria \\ \{eiter, tkren, patrik\}@kr.tuwien.ac.at
}

\begin{abstract}
With the advent of publicly available geospatial data, ontology-based data access (OBDA) over spatial data has gained increasing interest. Spatiorelational DBMSs are used to implement geographic information systems (GIS) and are fit to manage large amounts of data and geographic objects such as points, lines, polygons, etc. In this paper, we extend the Description Logic DL-Lite with spatial objects and show how to answer spatial conjunctive queries (SCQs) over ontologies - that is, conjunctive queries with point-set topological relations such as next and within-expressed in this language. The goal of this extension is to enable an off-the-shelf use of spatio-relational DBMSs to answer SCQs using rewriting techniques, where data sources and geographic objects are stored in a database and spatial conjunctive queries are rewritten to SQL statements with spatial functions. Furthermore, we consider keyword-based querying over spatial OBDA data sources, and show how to map queries expressed as simple keyword lists describing objects of interest to SCQs, using a meta-model for completing the SCQs with spatial aspects. We have implemented our lightweight approach to spatial OBDA in a prototype and show initial experimental results using data sources such as Open Street Maps and Open Government Data Vienna from an associated project. We show that for real-world scenarios, practical queries are expressible under meta-model completion, and that query answering is computationally feasible.
\end{abstract}

\section{Introduction}

By the ever increasing availability of mobile devices, location-aware search providers are becoming increasingly commonplace. Search providers (e.g., Google Maps http://maps.google.com/or Nokia Maps http://here.net) offer the possibility to explore their surroundings for desired locations, also called points-of-interest (POIs), but usually miss the possibility to express spatial relations (e.g., next and within). For an expressive location-aware search, the combination of Semantic Web techniques and spatial data processing (with spatial relations) is appropriate, given they provide a data backbone for spatial and taxonomic information to query semantically-enriched POIs.

To realize location-aware semantic search support, one needs to capture categories of POIs (e.g., Italian restaurant), their relations to additional qualitative attributes (e.g.,

\footnotetext{
* Supported by the Austrian Research Promotion Agency (FFG) project P828897, and the Austrian Science Fund (FWF) project P20840.
} 


\begin{tabular}{|c|c|c|}
\hline Shop $\sqsubseteq$ SpatialFeat & hasOp $\sqsubseteq$ hasQVal & $O p \sqsubseteq Q V a l$ \\
\hline$\exists$ hasQVal ${ }^{-} \sqsubseteq$ SpatialFeat & Shop $\sqsubseteq \exists h a s O p$ & Wlan $\sqsubseteq Q$ Val \\
\hline Park $\sqsubseteq$ SpatialFeat & $\exists h a s O p^{-} \sqsubseteq O p$ & GuestGarden $\sqsubseteq Q$ Val \\
\hline Supermarket $\sqsubseteq$ Shop & $Q V a l \sqsubseteq \exists h a s Q V a l$ & SpatialFeat $\sqsubseteq \neg$ Geometry \\
\hline
\end{tabular}

Fig. 1. Ontology with integrated meta-model (TBox excerpt; role names start lowercase)

having a guest garden). Further, one needs to capture the spatial relations between POIs (e.g., located inside a park). For modeling and interpreting a user's intention, it seems suggestive to use ontology languages and associated reasoning services. However, for spatial aspects we need to extend or combine them with spatial data reasoning. Furthermore, we must respect that ordinary users of location-aware search need a plain query interface; they are not experts in query languages, and an interface to express search intentions by lists of keywords in a Google-like manner would be desirable.

However, we face several obstacles for a seamless keyword-based querying and integration of geospatial data sources and ontologies. First, for a meaningful search result, we need to consider data obtained by integrating multiple data sources, which may be provided by autonomous vendors in heterogeneous formats (e.g., OpenStreetMap or Open Government Data data, a restaurant guide, etc). Using various data sources of substantial size gives the opportunity to find intended POIs, which may fall into multiple concepts ranging from rather generic to more detailed ones such as "restaurant" vs. "pizzeria." Moreover, we can exploit the structure of the taxonomic information that is implicit in the data sources by making it concrete in an ontology. Such ontology-based data access can be used to answer broad queries like "restaurants with Italian Cuisine," that should return pizzerias, trattorias, and osterias.

Second, from keyword-based input, we must generate meaningful formal queries to an ontology. In that, we must respect that the users may have no prior knowledge of the domain. Thus, we must be able to recognize and generate relevant combinations of possible keywords according to the ontology that represents the domain.

Third, as we query mainly spatial data, we need to capture spatial relations between different spatial objects and give users the possibility to use a fixed set of keywords to express them. For spatial querying answering, we must define an appropriate semantics and provide techniques that combine spatial with ontological query answering.

Fourth, a lot of research has been put into efficient query answering techniques over lightweight ontology languages, such as the DL-Lite family [7]. Conjunctive query (CQ) evaluation over DL-Lite ontologies can be delegated, by first-order query rewriting, to a Relational Database Management System (RDBMS), which facilitates scalable query processing. To secure this for an extension with spatial reasoning, the first-order rewritability of the latter is desirable. Furthermore, as first-order rewritings of queries might get prohibitively large in general (a known feature), also issues of manageable query generation from keywords must be respected.

We address the above issues with the following approach outlined in a nutshell.

- Various data sources are integrated via a global schema represented by an DL-Lite ${ }_{R}$ ontology that is enriched with spatial information. The ontology-based knowledge base 
(KB) is separated into a TBox, an ABox with normal individuals and a spatio-relational database with spatial objects. We apply a mild extension to DL-Lite ${ }_{R}$ by associating individuals to spatial objects by a predefined binding. A preprocessor creates this binding using a domain-specific heuristic (which is not considered here).

- The enriched ontology can be accessed, at the system level, by spatial conjunctive queries $(S C Q)$, which extend conjunctive queries with spatial predicates (e.g. intersects). In such queries, individuals can be located with spatial objects whose relationships are determined. By rewriting techniques, and in exploiting the PerfectRef algorithm [7], SCQs can be rewritten to a union of conjunctive queries (UCQ). Under certain syntactic conditions, a 2-stage evaluation-evaluation of the ontology part of the query (over the ABox, which is stored in an RDBMS) followed by filtering via spatial conditions-is possible, which makes this approach attractive for practical realization.

- For keyword-based query answering, concepts of the ontology are labeled with keywords. On query evaluation, the keywords which the user enters are mapped to concepts and roles from the ontology; an auto-completion service aids the user to compensate lack of domain knowledge. Based on the keyword structure, a feasible CQ is generated and extended with spatial predicates to SCQs; in that, we use a specific meta-model that is stored in the ontology. Fig. 1 shows an excerpt of the ontology; the concept SpatialFeat intuitively says that the individual has spatial features, which is extended by the subroles of has $Q \mathrm{Val}$ with qualitative values, which are asserted to subconcepts of $Q \mathrm{Val}$. Furthermore, the individual is represented by a geometry, asserted to subconcepts of Geometry. However, also normal role assertions for qualitative attributes are considered (e.g., a restaurant with a guest garden).

We have implemented this approach in an experimental prototype, which is part of a more extensive system for smart, semantically enriched route planning system (MyITS, http://myits.at/) over real world data sources such as OpenStreetMap (OSM), Open Government Data (OGD) of Vienna, and the Falter restaurant guide for Vienna. The data sources are integrated by a global schema represented by an ontology ex-

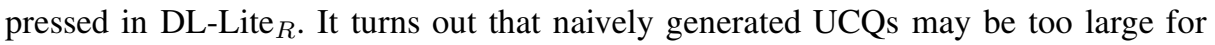
execution on conventional RDBMS. We thus improved our approach by exploiting the structure of the TBox in an optimized generation of queries from keyword, to eventually obtain smaller UCQs. First experiments show that this approach is feasible in a real-world scenario. Furthermore, we show that the optimizations described are important for feasibility. An extended version of this paper provides more details that are omitted for space reasons 1

\section{Preliminaries}

We adopt DL-Lite ${ }_{R}$ [7] as the underlying ontological language and introduce an approach in which the FO-rewriting of PerfectRef (see [7] and [6] for details) is strictly separated from spatial querying. As a result of this separation, we only allow spatial predicates (e.g., Contains) on the top level of the query structure. Regarding the semantics, we following partly the ideas of [15], but focus primarily on query answering (not solely satisfiability). Furthermore, we use a different notion for spatial relations.

\footnotetext{
${ }^{1}$ http://www.kr.tuwien.ac.at/staff/patrik/ESWC2013Ext.pdf
} 
Point-Set Topological Relations. We follow the point-set topological relation model in [13], where spatial relations are defined in terms of pure set theoretic operations. The realization of spatial objects is based on a set $P_{E} \subseteq \mathbb{R}^{2}$ of points in the plane; the (names of) spatial objects themselves are in a set $\Gamma_{S}$. While the set of points for a spatial object $s$ is infinite (unless it is a point), it can be finitely defined by an associated admissible geometry $g(s)$. The geometries are defined by sequences $p=\left(p_{1}, \ldots, p_{n}\right)$ of points that induce a point $(n=1)$, a line segment $(n=2)$, a line $(n>2)$, or a polygon. All points used for admissible geometries are from a finite set $P_{F} \subseteq P_{E}$ of points.

Spatio-relational Database. Thus, we define a spatio-relational database over $\Gamma_{S}$ as a pair $\mathcal{S}=\left(P_{F}, g\right)$ of a point set $P_{F} \subseteq \mathbb{R}^{2}$ and a mapping $g: \Gamma_{S} \rightarrow \bigcup_{i>1} P_{F}{ }^{i}$.

The extent of a geometry $p$ (full point set) is given by the function points $(p)$ and is a (possibly infinite) subset of $P_{E}$. For a spatial object $s$, we let points $(s)=\operatorname{points}(g(s))$. We need points to evaluate the spatial relations of two spatial objects via their respective geometries. For our spatio-thematic KBs, we consider the following types of admissible geometries $p$ over $P_{F}$ (with their representation), and let $P_{E}=\bigcup_{s \in \Gamma_{S}} \operatorname{points}(s)$ : a

- point is a sequence $p=\left(p_{1}\right)$, where points $\left(p_{1}\right)=\left\{p_{1}\right\}$;

- line segment is a sequence $p=\left(p_{1}, p_{2}\right)$, and points $(p)=\left\{\alpha p_{1}+(1-\alpha) p_{2} \mid \alpha \in\right.$ $\mathbb{R}, 0 \leq \alpha \leq 1\}$

- line is a sequence $p=\left(p_{1}, \ldots, p_{n}\right)$ of line segments $\left(p_{i}, p_{i+1}\right), 1 \leq i<n$, the first $\left(p_{1}, p_{2}\right)$ and last $\left(p_{n-1}, p_{n}\right)$ segments do not share an end-point, and points $(p)=$ $\bigcup_{i=1}^{n-1}$ points $\left(p_{i}\right)$;

- polygon is like a line but $\left(p_{1}, p_{2}\right)$ and $\left(p_{n-1}, p_{n}\right)$ share an end point; we have points $(a)=\bigcup_{i=1}^{n-1} \operatorname{points}\left(p_{i}\right) \cup \operatorname{int}\left(l_{c}\right)$, where $\operatorname{int}\left(l_{c}\right)$ is the interior built from the separation of $P_{E}$ by $p$ into two disjoint regions.

Some $s \in \Gamma_{S}$ may serve to define via $g$ a bounding box. We omit more complex geometries like areas or polygons with holes. Based on points $(x)$, we can define the spatial relation of point-sets in terms of pure set operations:

- Equals $(x, y):$ points $(x)=$ points $(y)$ and NotEquals $(x, y):$ points $(x) \neq$ points $(y)$;

- Inside $(x, y):$ points $(x) \subseteq$ points $(y)$ and Outside $(x, y): \operatorname{points}(x) \cap$ points $(y)=\emptyset$;

- Intersect $(x, y): \operatorname{points}(x) \cap$ points $(y) \neq \emptyset$ and $N \operatorname{ext} T o(x, y): b(x) \cap b(y) \neq$ $\emptyset$, where $b(z)=\left\{a \in P_{E} \mid \operatorname{dist}(a, \operatorname{points}(z)) \leq d_{B}\right\}$ for a distance function dist $: \mathbb{R}^{2} \rightarrow \mathbb{R}_{0}^{+}$and a distance value $d_{B} \in \mathbb{R}$.

Now for any spatial relation $S\left(s, s^{\prime}\right)$ and $s, s^{\prime} \in \Gamma_{S}$, holds on a spatio-relational DB $\mathcal{S}$, written $\mathcal{S} \models S\left(s, s^{\prime}\right)$, if $S\left(g(s), g\left(s^{\prime}\right)\right.$ evaluates to true. Relative to points and dist (and $\left.d_{B}\right)$, this is easily captured by a first-order formula over $\left(\mathbb{R}^{2}, \leq\right)$, and with regard to geo-spatial RDBMS trivially first-order expressible.

Note that the space model of [13] differs from the more detailed 9-Intersection model (DE-9IM) of [10], which considers strict separation of the interior and object boundary; this leads to 9 instead of 5 spatial relations. We also omit spatial predicates in the signature, assuming a "standard" point-set interpretation of the spatial-relations [13]. Our approach is modular and flexible enough to allow further relations (e.g., connects) or use other interpretations such as DE-9IM. 
Syntax and Semantics of DL-Lite ${ }_{R}$. We recall the definitions from [7]. Consider a vocabulary of individual names $\Gamma_{I}$, atomic concepts $\Gamma_{C}$, and atomic roles $\Gamma_{R}$. Given atomic concepts $A$ and atomic roles $P$, we define basic concepts $B$ and basic roles $R$, complex concepts $C$ and complex role expressions $E$, and $P^{-}$be the inverse of $P$ as

$$
B::=A|\exists R \quad C::=B| \neg B \quad R::=P\left|P^{-} \quad E::=R\right| \neg R .
$$

A DL-Lite ${ }_{R}$ knowledge base is a pair $\mathcal{K}=(\mathcal{T}, \mathcal{A})$ where the TBox $\mathcal{T}$ consists of a finite set of inclusion assertions of the form $B \sqsubseteq C$ and $R \sqsubseteq E$, and the ABox $\mathcal{A}$ is a finite set of membership assertions on atomic concepts and on atomic roles of the form $A(a)$ and $P(a, b)$, where $a$ and $b$ are individual names.

The semantics of DL-Lite ${ }_{R}$ is given in terms of FO interpretations $\mathcal{I}=\left(\Delta^{\mathcal{I}},{ }^{\mathcal{I}}\right)$, where $\Delta^{\mathcal{I}}$ is a nonempty domain and ${ }^{\mathcal{I}}$ an interpretation function such that $a^{\mathcal{I}} \in \Delta^{\mathcal{I}}$ for all $a \in \Gamma_{I}, A^{\mathcal{I}} \subseteq \Delta^{\mathcal{I}}$ for all $A \in \Gamma_{C}, P^{\mathcal{I}} \subseteq \Delta^{\mathcal{I}} \times \Delta^{\mathcal{I}}$ for all $P \in \Gamma_{R}$, and $\left(P^{-}\right)^{\mathcal{I}}=\left\{\left(a_{2}, a_{1}\right) \mid\left(a_{1}, a_{2}\right) \in P^{\mathcal{I}}\right\} ;(\exists R)^{\mathcal{I}}=\left\{a_{1} \mid \exists a_{2} \in \Delta^{\mathcal{I}}\right.$ s.t. $\left.\left(a_{1}, a_{2}\right) \in R^{\mathcal{I}}\right\} ;$ $(\neg B)^{\mathcal{I}}=\Delta^{\mathcal{I}} \backslash B^{\mathcal{I}}$; and $(\neg R)^{\mathcal{I}}=\Delta^{\mathcal{I}} \times \Delta^{\mathcal{I}} \backslash R^{\mathcal{I}}$.

The notions of satisfaction of inclusion axioms and assertions, TBox and ABox resp. knowledge base is as usual, as well as logical implication; both are denoted with $\models$. We assume the unique name assumption holds for different individuals and values.

Checking satisfiability of DL-Lite ${ }_{R}$ ontologies is first-order (FO) rewritable [7], i.e., for all $\mathcal{T}$, there is a Boolean FO query $Q_{\mathcal{T}}$ (constructible from $\mathcal{T}$ ) s.t. for every $\mathcal{A}, \mathcal{T} \cup \mathcal{A}$ is satisfiable iff $D B(\mathcal{A}) \not \models Q_{\mathcal{T}}$, where $D B(\mathcal{A})$ is the least Herbrand model of $\mathcal{A}$.

\section{DL-Lite $_{R}(S)$}

In this section, we extend DL-Lite ${ }_{R}$ with spatial objects to DL-Lite ${ }_{R}(S)$. We present its syntax and semantics, a transformation of to DL-Lite, and show that satisfiability and conjunctive query answering over DL-Lite ${ }_{R}(S) \mathrm{KBs}$ are FO-rewritable.

Syntax. Let $\Gamma_{S}$ and $\Gamma_{I}$ be pairwise disjoint sets as defined above. A spatio-thematic knowledge base (KB) is defined as $\mathcal{L}_{\mathcal{S}}=\langle\mathcal{T}, \mathcal{A}, \mathcal{S}, \mathcal{B}\rangle$, where $\mathcal{T}$ (resp. $\mathcal{A}$ ) is defined as a DL-Lite $R$ TBox (resp. ABox), $\mathcal{S}$ is a spatio-relational database, and $\mathcal{B} \subseteq \Gamma_{I} \times \Gamma_{S}$ is a partial function called the binding from $\mathcal{A}$ to $\mathcal{S}$, similar to [15]; we apply a mild extension to DL-Lite ${ }_{R}$ by associating individuals from $\mathcal{A}$ to spatial objects from $\mathcal{S}$.

We assume $\mathcal{B}$ to be already given. Furthermore, we extend DL-Lite ${ }_{R}$ with the ability to specify the localization of a concept. For this purpose, we extend the syntax with

$$
C::=B|\neg B|(\operatorname{loc} A) \mid\left(l o c_{s} A\right), \quad s \in \Gamma_{S},
$$

where $A$ is an atomic concept in $\mathcal{T}$; intuitively, (loc $A$ ) is the set of individuals in $A$ that can have a spatial extension, and $\left(l o c_{s} A\right)$ is the subset which have extension $s$.

Semantics. Our aim is to give a semantics to the localization concepts $(l o c A)$ and $\left(l o c_{s} A\right)$ such that a $\mathrm{KB} \mathcal{L}_{\mathcal{S}}=\langle\mathcal{T}, \mathcal{A}, \mathcal{S}, \mathcal{B}\rangle$ can be readily transformed into an ordinary DL-Lite $_{R} \mathrm{~KB} \mathcal{K}_{\mathcal{S}}=\left\langle\mathcal{T}^{\prime}, \mathcal{A}^{\prime}\right\rangle$, using concepts $C_{\mathcal{S}_{\mathcal{T}}}$ and $C_{s}$ for individuals with some spatial extension resp. located at $s$. Note that $C_{\mathcal{S}_{\mathcal{T}}}$ cannot be forced to be the union of all $C_{s}$, as this would introduce disjunction (this hinders the passing from the open to the closed world assumption, which is important for the FO-rewriting of DL-Lite). 
An $\left(\right.$ DL-Lite $\left._{R}\right)$ interpretation of $\mathcal{L}_{\mathcal{S}}$ is a structure $\mathcal{I}=\left\langle\Delta^{\mathcal{I}},{ }^{\mathcal{I}}, b^{\mathcal{I}}\right\rangle$, where $\left\langle\Delta^{\mathcal{I}},{ }^{\mathcal{I}}\right\rangle$ is an interpretation of $\langle\mathcal{T}, \mathcal{A}\rangle$, and $b^{\mathcal{I}} \subseteq \Delta^{\mathcal{I}} \times \Gamma_{S}$ is a partial function that assigns some individuals a location, such that for every $a \in \Gamma_{I},(a, s) \in \mathcal{B}$ implies $b^{\mathcal{I}}\left(a^{\mathcal{I}}\right)=s$.

We extend the semantics of the previous section with $(\operatorname{loc} A),\left(\operatorname{loc}_{s} A\right)$, where $A$ is an atomic concept in $\mathcal{T}$ :

$$
\begin{aligned}
& (\operatorname{loc} A)^{\mathcal{I}} \supseteq\left\{e \in \Delta^{\mathcal{I}} \mid e \in A^{\mathcal{I}} \wedge \exists s \in \Gamma_{S}:(e, s) \in b^{\mathcal{I}}\right\}, \\
& \left(\operatorname{loc}_{s} A\right)^{\mathcal{I}}=\left\{e \in \Delta^{\mathcal{I}} \mid e \in A^{\mathcal{I}} \wedge(e, s) \in b^{\mathcal{I}}\right\} .
\end{aligned}
$$

The interpretation of complex concepts, satisfaction, etc. is then as usual. For example, $A \sqsubseteq\left(l o c_{s} A\right)$ expresses that all individuals in $A$ are located at $s ; B \sqsubseteq(\operatorname{loc} A)$ states that individuals in $B$ can have a location if they are in $A$.

Transformation to DL-Lite $R_{R}$. Let $C_{\mathcal{S}_{\mathcal{T}}}$ and $C_{s}$, for every $s \in \Gamma_{S}$, be fresh concepts. We transform $\mathcal{L}_{\mathcal{S}}$ to $\mathcal{K}_{\mathcal{S}}=\left\langle\mathcal{T}^{\prime}, \mathcal{A}^{\prime}\right\rangle$, where $\mathcal{T}^{\prime}=\tau(\mathcal{T}) \cup \mathcal{T}_{\mathcal{S}}$ and $\mathcal{A}^{\prime}=\tau(\mathcal{A}) \cup \mathcal{A}_{\mathcal{B}}$, and

- $\tau(X)$ replaces each occurrence of $(l o c A)$ and $\left(l o c_{s} A\right)$ in $X$ with $C_{\mathcal{S}_{\mathcal{T}}} \sqcap A$ and $C_{s} \sqcap A$, respectively, and splits $\sqcap$ up;

- $\mathcal{T}_{\mathcal{S}}$ represents generic localization information via concepts, and contains the axiom $C_{s} \sqsubseteq C_{\mathcal{S}_{\mathcal{T}}}$, and the constraints $C_{s} \sqsubseteq \neg C_{s^{\prime}}$ for all $s \neq s^{\prime} \in \Gamma_{S}$;

- $\mathcal{A}_{\mathcal{B}}$ represents the concrete bindings between $\mathcal{A}$ and $\Gamma_{S}$, and for every $(a, s) \in \mathcal{B}$, we add $C_{s}(a)$ in $\mathcal{A}_{\mathcal{B}}$. Note that we do not assert $\neg C_{s}(a)$ for $(a, s) \notin \mathcal{B}$, keeping the open world assumption for bindings.

For example, let $A$ (resp. $C_{\mathcal{S}_{\mathcal{T}}}$ ) be the concept Park (resp. SpatialFeat), cp be the spatial object of "City Park," and the polygon poly_cp representing cp's spatial extend. The KB has the assertions Park $\sqsubseteq$ (loc Park), CityParkCafe $\sqsubseteq\left(\right.$ loc $_{c p}$ Park $)$, and CityParkCafe (c). Then, the transformation produces Park $\sqsubseteq$ (SpatialFeat $\sqcap$ Park), CityParkCafe $\sqsubseteq\left(C_{\text {poly_cp }} \sqcap\right.$ Park $), C_{\text {poly_cp }} \sqsubseteq$ SpatialFeat, and $C_{\text {poly_cp }}(c p)$.

Note that $\mathcal{K}_{\mathcal{S}}$ is indeed a DL-Lite ${ }_{R}$ ontology, by the syntactic restrictions on localization concepts. It is not hard to verify that the models of $\mathcal{L}_{\mathcal{S}}$ and $\mathcal{K}_{\mathcal{S}}$ with the same domain $\left(\Delta^{\mathcal{I}}=\Delta^{\mathcal{I}^{\prime}}\right)$ coincide on common concepts and roles as follows: (i) if $\mathcal{I} \models \mathcal{L}_{\mathcal{S}}$, then $\mathcal{I}^{\prime} \models \mathcal{K}_{\mathcal{S}}$ where $C_{s}^{\mathcal{I}^{\prime}}=\left\{e \in \Delta^{\mathcal{I}} \mid(e, s) \in b^{\mathcal{I}}\right\}, C_{\mathcal{S}_{\mathcal{T}}}^{\mathcal{I}^{\prime}}=\bigcup_{s \in \Gamma_{S}} C^{\mathcal{I}^{\prime}}$ $\left(=\operatorname{dom}\left(b^{\mathcal{I}}\right)\right)$; conversely, (ii) if $\mathcal{I}^{\prime} \models \mathcal{K}_{\mathcal{S}}$, then $\mathcal{I}=\mathcal{L}_{\mathcal{S}}$ where $b^{\mathcal{I}}=\left\{(e, s) \mid e \in C_{s}^{\mathcal{I}^{\prime}}\right\}$ and $(\text { loc } A)^{\mathcal{I}}=C_{\mathcal{S}_{\mathcal{T}}}^{\mathcal{I}^{\prime}} \cap A^{\mathcal{I}^{\prime}}$. As an easy consequence of this correspondence, we obtain:

Proposition 1. Satisfiability checking and CQ answering for ontologies in DL-Lite $R(S)$ is FO-rewritable.

As the models of $\mathcal{L}_{\mathcal{S}}$ and $\mathcal{K}_{\mathcal{S}}$ correspond, we can check satisfiability on $\mathcal{K}_{\mathcal{S}}$, i.e., a standard DL-Lite ${ }_{R} \mathrm{~KB}$. An ontology CQ $q$ over $\mathcal{L}_{\mathcal{S}}$ is easily rewritten to a CQ over $\mathcal{K}_{\mathcal{S}}$.

\section{Query Answering in DL-Lite ${ }_{R}(S)$}

We next define spatial conjunctive queries (SCQ) over $\mathcal{L}_{\mathcal{S}}=\langle\mathcal{T}, \mathcal{A}, \mathcal{S}, \mathcal{B}\rangle$. Such queries may contain ontology and spatial predicates. Formally, an SCQ $q(\mathbf{x})$ is a formula

$$
Q_{O_{1}}(\mathbf{x}, \mathbf{y}) \wedge \cdots \wedge Q_{O_{n}}(\mathbf{x}, \mathbf{y}) \wedge Q_{S_{1}}(\mathbf{x}, \mathbf{y}) \wedge \cdots \wedge Q_{S_{m}}(\mathbf{x}, \mathbf{y})
$$

where $\mathbf{x}$ are the distinguished variables and $\mathbf{y}$ are either non-distinguished (bound) variables or individuals from $\Gamma_{I}$. Each $Q_{O_{i}}(\mathbf{x}, \mathbf{y})$ is an atom for $\mathcal{T}$ and of the form 
$A(z)$ or $P\left(z, z^{\prime}\right)$, with $z, z^{\prime}$ from $\mathbf{x} \cup \mathbf{y}$; the atoms $Q_{S_{j}}(\mathbf{x}, \mathbf{y})$ are over the vocabulary for the spatial relations in Sec. 2] and of the form $S\left(z, z^{\prime}\right)$, with $z, z^{\prime}$ from $\mathbf{x} \cup \mathbf{y}$.

For example, $q(x)=\operatorname{Playground}(x) \wedge W i \operatorname{thin}(x, y) \wedge \operatorname{Park}(y)$ is a SCQ which intuitively returns the playgrounds located in parks.

Semantics. Let $\mathcal{I}=\left\langle\Delta^{\mathcal{I}},{ }^{\mathcal{I}}, b^{\mathcal{I}}\right\rangle$ be an interpretation of $\mathcal{L}_{\mathcal{S}}$. A match for $q(\mathbf{x})$ in $\mathcal{I}$ is a function $\pi: \mathbf{x} \cup \mathbf{y} \rightarrow \Delta^{\mathcal{I}}$ such that $\pi(c)=c^{\mathcal{I}}$, for each constant $c$ in $\mathbf{x} \cup \mathbf{y}$, and for each $i=1, \ldots n$ and $j=1, \ldots, m$, (i) $\pi(z) \in A^{\mathcal{I}}$, if $Q_{O_{i}}(\mathbf{x}, \mathbf{y})=A(z)$; (ii) $\left(\pi(z), \pi\left(z^{\prime}\right)\right) \in$ $P^{\mathcal{I}}$, if $Q_{O_{i}}(\mathbf{x}, \mathbf{y})=P\left(z, z^{\prime}\right)$; and (iii) $\exists s, s^{\prime} \in \Gamma_{S}:(\pi(z), s) \in b^{\mathcal{I}} \wedge\left(\pi\left(z^{\prime}\right), s^{\prime}\right) \in$ $b^{\mathcal{I}} \wedge \mathcal{S} \models S\left(s, s^{\prime}\right)$, if $Q_{S_{j}}(\mathbf{x}, \mathbf{y})=S\left(z, z^{\prime}\right)$. That is, for spatial predicates individuals must have (unique) spatial extensions and the relationship between them must hold.

Then, a tuple $\mathbf{c}=c_{1}, \ldots, c_{k}$ over $\Gamma_{I}$ is an answer for $q(\mathbf{x})$ in $\mathcal{I}, \mathbf{x}=x_{1}, \ldots$, if $q(\mathbf{x})$ has some match $\pi$ in $\mathcal{I}$ such that $\pi\left(x_{i}\right)=c_{i}, i=1, \ldots, k$; furthermore, $\mathbf{c}$ is an answer for $q(\mathbf{x})$ over $\mathcal{L}_{\mathcal{S}}$, if it is an answer in every model $\mathcal{I}$ of $\mathcal{L}_{\mathcal{S}}$. The result of $q(\mathbf{x})$ over $\mathcal{L}_{\mathcal{S}}$, denoted $\operatorname{res}\left(q(\mathbf{x}), \mathcal{L}_{\mathcal{S}}\right)$, is the set of all its answers.

The semantic correspondence between $\mathcal{L}_{\mathcal{S}}$ and $\mathcal{K}_{\mathcal{S}}=\left\langle\mathcal{T}^{\prime}, \mathcal{A}^{\prime}\right\rangle$ guarantees that we can transform $q(\mathrm{x})$ into an equivalent query over $\mathcal{L}_{\mathcal{S}}{ }^{\prime}=\left\langle\mathcal{T}^{\prime}, \mathcal{A}^{\prime}, \mathcal{S}, \mathcal{B}\right\rangle$ by replacing each spatial atom $S\left(z, z^{\prime}\right)$ in $q(\mathbf{x})$ with

$$
\bigvee_{s, s^{\prime} \in \Gamma_{S}}\left(C_{s}(z) \wedge C_{s}\left(z^{\prime}\right) \wedge S\left(s, s^{\prime}\right)\right)
$$

The resulting formula is easily cast into form $u q(\mathbf{x})=q_{1}(\mathbf{x}) \vee \cdots \vee q_{l}(\mathbf{x})$, i.e., a union of CQs $q_{i}(\mathbf{x})$. The answers of $u q(\mathbf{x})$ in an interpretation $\mathcal{I}^{\prime}$ of $\mathcal{L}_{\mathcal{S}}{ }^{\prime}$ are the answers of all $q_{i}(\mathbf{x})$ in $\mathcal{I}^{\prime}$, and $\operatorname{res}\left(u q(\mathbf{x}), \mathcal{L}_{\mathcal{S}}{ }^{\prime}\right)$ is defined in the obvious way. We then can show:

Proposition 2. For every $S Q C q(\mathbf{x})$ over $\mathcal{L}_{\mathcal{S}}, \operatorname{res}\left(q(\mathbf{x}), \mathcal{L}_{\mathcal{S}}\right)=\operatorname{res}\left(u q(\mathbf{x}), \mathcal{L}_{\mathcal{S}}{ }^{\prime}\right)$.

Hence, answering SCQs in DL-Lite ${ }_{R}(S)$ ontologies is FOL-rewritable. In particular, for fixed $\mathcal{S}$, we can eliminate $S\left(s, s^{\prime}\right)$ from (2), which yields a pure ontology query. Alternatively, we can replace it with $S_{s, s^{\prime}}(z)$, where $S_{s, s^{\prime}}$ is a fresh concept, and add $C_{s} \sqsubseteq S_{s, s^{\prime}}$ to the TBox $\mathcal{T}^{\prime}$ iff $\mathcal{S} \models S\left(s, s^{\prime}\right)$, thus changing $\mathcal{S}$ more flexibly.

Spatial Conjunctive Query Evaluation. The above SCQ rewriting is exponential in the number of spatial atoms, which incurs limitations. However, if no bounded variables occur in spatial atoms, we can separate query answering into an ontology part and a spatial query part, which can be efficiently evaluated in two stages:

(1) evaluate the ontology part of the query $q(\mathbf{x})$ (i.e., drop all spatial atoms) over $\mathcal{L}_{\mathcal{S}}{ }^{\prime}$. For that we can apply the standard DL-Lite ${ }_{R}$ query rewriting of PerfectRef and evaluate the result over the ABox, stored in an RDBMS.

(2) filter the result of Step (1), by evaluating the formulas (2) on the bindings $\pi$ for the distinguished variables $\mathbf{x}$ (which are mapped to individuals). For that, retrieve in Step (1) also all instances of $C_{s}$, for all $s \in \Gamma_{S}$.

Step (2) amounts to computing a spatial join $\bowtie_{S}$, for which (at least) different evaluation strategies exist. One strategy, denoted as $O_{D}$, relies entirely on the functions of a spatial-extended RDBMS. The other, denoted as $O_{I}$, relies on an internal evaluation of $\bowtie_{S}$, i.e., spatial relations, where the intermediate results are kept in-memory.

We have considered both strategies, restricting to acyclic queries (i.e., the query hypergraph is tree-shaped; see e.g. [12] for a definition). For such queries, join trees can be built, which can be processed in a bottom up manner. In doing so, we distinguish 
between ontology and spatial nodes, and actually interleave the DL-Lite ${ }_{R}$ query rewriting (Step (10) and spatial join checking (Step (2)). For space reasons, we omit details.

Note that for strategy $O_{D}$, we rewrite the spatial atoms (Contains, Within, etc.) directly to corresponding functions (cf. [8] for details) of the spatial-extension of the RDBMS. The different strategies noticeably affect the performance (see Sec. 8).

\section{From Keywords to Spatial Conjunctive Queries}

In this section, we provide the details for the generation of SCQ from a valid sequence of keywords; We shall consider in the next section how such sequences are obtained in a controlled way, by automatic completion and checking keyword combinations.

We assume an ontology $O_{U}$, which has an associated meta-model for structuring the query generation (described below). The generation is realized in three steps. First, the keywords are mapped to concepts from $O_{U}$ and to spatial predicates. Then, a set of completion rules (which regard the meta-model) is applied to the resulting sequence of atomic formulas. Finally the completed sequence is converted into a SCQ.

We assume that spatio-thematic KBs are labeled, i.e., they are of the form $\mathcal{L}_{\mathcal{S}}=$ $\langle\mathcal{T}, \mathcal{A}, \mathcal{S}, \mathcal{B}, \mathcal{N}\rangle$, where $\mathcal{N}$ is a set of textual labels representing keywords. The labels of $\mathcal{N}$ are assigned by rdfs: label to the concepts of $\mathcal{T}$. Multiple labels can be assigned to a single element, which allows to have synonyms. Further, translations for keywords in different languages can be enabled by the assignments.

Meta-model for Structured Query Generation. We require on the top level of the ontology in use a strict separation of the concepts for spatial features SpatialFeat (e.g., Park, Restaurant, etc.), qualitative values $Q$ Val (e.g., operator $O p$, Cuisine, etc.), and Geometry (e.g., Point, Polygon, etc.). Since our approach is designed to query spatial objects, every query has to be related to some SpatialFeat, which is extended by the subroles of has $Q \mathrm{Val}$ with qualitative values (asserted to $Q \mathrm{Val}$ ) and is represented by the role hasGeometry as a geometry (asserted to Geometry). By this separation on the top level (which also exists in GeoOWL http: / /www.w3.org/2005/Incubator/ geo/XGR-geo/), we have a meta-model, which is then used for the generation of "meaningful" queries. Any ontology used with our approach has to be structured according to the meta-model. Fig. 1 shows some axioms of the meta-model for a specific ontology.

Generation of SCQs from Keywords. The automatic completion and combination step produces a set of valid keyword sequences, from which one sequence $K=\left(k_{1}\right.$, $k_{2}, \ldots, k_{n}$ ) is chosen (unless the user determines one). Each keyword $k_{i}$ represents either a concept or a spatial predicate. We must connect all $k_{i}$ according to the metamodel to obtain SCQs, which then evaluate to spatial objects.

The rewriting of $K$ to a SCQ $Q$ is based on three steps that resemble a transducer with a context-free (left-recursive) grammar and a set of completion rules. The latter are important, because even if the transducer generates syntactically correct queries, their results might not consist of spatial objects. E.g., we could have a query ItalianCuisine $(x)$, but the results $R=($ pizza, pasta, ... $)$ could not be located on a map. Therefore, we have to extend the query as follows: Restaurant $(x) \wedge$ hasCuisine $(x, y) \wedge$ ItalianCuisine $(y)$. 
Table 1. Completion rules; the result of rules (R1)-(R 4 is denoted as subquery (SQ)

(R1) If $C_{1} \sqsubseteq$ SpatialFeat and $C_{2} \sqsubseteq$ QualAttribute rewrite to $\left(C_{1}\right.$ hasQVal $\left.C_{2}\right)$;

(R2) If $C_{1} \sqsubseteq$ SpatialFeat, $C_{2} \sqsubseteq$ QualAttribute, $C_{3} \sqsubseteq$ QualAttribute rewrite to $\left(\left(C_{1}\right.\right.$ hasQVal $\left.C_{2}\right)$ hasQVal $\left.C_{3}\right)$;

(R3) If $C_{1} \sqsubseteq$ QualAttribute rewrite to (SpatialFeat hasQVal $C_{1}$ );

(R4) If $C_{1} \sqsubseteq$ QualAttribute and $C_{2} \sqsubseteq$ QualAttribute rewrite to ( $\left(\right.$ SpatialFeat hasQVal $\left.C_{1}\right)$ hasQVal $\left.C_{2}\right)$;

(R5) If $E_{1} \sqsubseteq$ SpatialFeat or $E_{1}$ is a SQ, $E_{2} \sqsubseteq$ SpatialFeat or $E_{2}$ is SQ, and $S$ is a spatial predicate rewrite to $\left(\left(E_{1}\right) S E_{2}\right)$

(R6) If $E_{1} \sqsubseteq$ SpatialFeat or $E_{1}$ is a SQ, and $E_{2} \sqsubseteq$ SpatialFeat or $E_{2}$ is SQ rewrite to $\left(\left(E_{1}\right)\right.$ NextTo $\left.E_{2}\right)$;

In the following, we describe the three steps in the rewriting of $K$ in detail:

(1) We obtain a new sequence $K^{\prime}$ from the sequence $K$ by replacing every keyword with either a concept from $\mathcal{T}$ or a predefined spatial predicate. We check whether the keywords are associated to a concept in $\mathcal{N}$, otherwise we ignore it.

(2) We apply the completion rules in Table 1 on $K^{\prime}$ in a left-to-right order until no rules are applicable, resulting in a sequence $K^{\prime \prime}$.

(3) We generate the query $q(\mathbf{x})$ from $K^{\prime \prime}$ according to the function

$$
f\left(K^{\prime \prime}\right)=\left(\cdots\left(\left(C_{1}\left(x_{1}\right) \wedge E_{1,1}\left(x_{1}, y_{1}\right) \wedge E_{1,2}\left(y_{1}\right)\right) \wedge \chi_{2}\right) \wedge \cdots\right) \wedge \chi_{n}
$$

where $\chi_{i}=E_{i, 1}\left(\vartheta\left(E_{i-1,1}\right), y_{i}\right) \wedge E_{i, 2}\left(y_{i}\right)$ and $C_{1}$ is a concept atom; each $E_{i, 1}$ is either empty, a role atom, or a spatial atom, and each $E_{i, 2}$ is either empty or a concept atom; $\vartheta\left(E_{i, 1}\right)$ is $x_{i}$ if $E_{i, 1}$ is a spatial atom, and $x_{i-1}$ if $E_{i, 1}$ is a role atom. These assignments ensure that the spatial atoms are always related to the top concept, while role atoms are related to the next level in the query tree.

After these steps, we obtain a valid SPQ $q(\mathbf{x})$ for query evaluation (Sec. 4). For rules (R2) - (R 4), Table 1 shows in fact a simplified version, as they could be extended to arbitrary sequences of QualAttributes. Furthermore, rule (R6) defines a default relationship, if two spatial features are queried. Rewriting them to a simple conjunction between $C_{1}(x)$ and $C_{2}(x)$ would often lead to empty results, as two identical objects assigned to different concepts do not often exist within geospatial data sources.

Example 1. Given the keywords $K=$ (italian cuisine, non-smoking, in, park), we apply the first step, where we replace every $k_{i}$ with an associated concept $C_{i}$ from $\mathcal{N}: K^{\prime}=($ ItalianCuisine, NonSmoking, Within, Park $)$. In the second step we apply the completion rules to obtain $K^{\prime \prime}=((($ SpatialFeat hasQVal ItalianCuisine $)$ hasQVal NonSmoking) Within Park). Finally we get a SCQ $q\left(x_{1}, x_{2}\right)=f\left(K^{\prime \prime}\right)$ with

$\operatorname{SpatialFeat}\left(x_{1}\right) \wedge \operatorname{has} Q \operatorname{Val}\left(x_{1}, y_{1}\right) \wedge \operatorname{ItalianCuisine}\left(y_{1}\right) \wedge \operatorname{has} Q \operatorname{Val}\left(x_{1}, y_{2}\right) \wedge$ $\operatorname{NonSmoking}\left(y_{2}\right) \wedge \operatorname{Within}\left(x_{1}, x_{2}\right) \wedge \operatorname{Park}\left(x_{2}\right)$.

\section{Generating Keyword Sequences}

Since our approach is designed to have a single text-field for the keyword entries, we aim to provide fast automatic completion, keywords detection, and keyword combination functions. If a user enters keywords on a user interface (UI), we guide her by 
automatic completion and by showing possible combinations compliant with the ontology. For that, we must take the structure of the KB into account. Furthermore, as many combinations may be compliant, a selection of "relevant" combinations must be provided.

As the need for very low response time (e.g., below 100ms) makes on-demand calculation from the $\mathrm{KB}$ infeasible, a prefix index is created offline that stores all possible prefixes for a label of $\mathcal{N}$. It amounts to a function $f_{P}(e)$ which maps a string $e$ to all possible labels of $\mathcal{N}$, such that $\bigcup_{n \in \mathcal{N}}(\operatorname{Pref}(e) \subseteq \operatorname{Pref}(n))$.

For example, the labels $\mathcal{N}=\{$ pub, public,park $\}, f_{P}$ map $p$, pub, and park as follows: $\{p\} \rightarrow\{$ park, pub, public $\},\{p u\} \rightarrow\{$ pub, public $\},\{$ park $\} \rightarrow\{$ park $\}$.

Syntactic Connectivity of Concepts. As multiple keywords are entered, we need to determine which concepts are connectable. We use a notion of syntactic connectivity $\mathbf{C}$ based on the syntactic structure of the KB, which captures the connection between two concepts through subconcepts and roles, but also through a common subsumer. For two concepts, we follow the inclusion assertion and check whether they share a common subsumer denoted as $C_{S}$, excluding the top concept. As the KB is based on DL-Lite ${ }_{R}$, we can capture the following inclusion assertions: (i) concept inclusion $M_{C}: C_{1} \sqsubseteq C_{2}$, role hierarchies $M_{H}: R_{1} \sqsubseteq R_{2}$; (ii) role membership which covers the range (resp. domain) of a role as $M_{R}: \exists R^{-} \sqsubseteq C$ (resp. $M_{D}: \exists R \sqsubseteq C$ ); and (iii) mandatory participation $M_{P}: C \sqsubseteq \exists R$. We deliberately do not consider disjoint concepts as $C_{1} \sqsubseteq \neg C_{2}$ in the inclusions, and distinguish direct and indirect connections between two concepts.

A direct connection between concepts $C_{A}$ and $C_{B}$ exists, denoted $\phi_{D}\left(C_{A}, C_{B}\right)$, if a sequence $C_{A} \rightarrow_{M} \exists R_{1} \rightarrow_{M} C_{1} \rightarrow_{M} \exists R_{1} \ldots C_{n} \rightarrow_{M} \exists R_{n} \rightarrow_{M} C_{B}$ exists, where $M=M_{D} \cup M_{H} \cup M_{C} \cup M_{R} \cup M_{P}$. Furthermore, an indirect connection between $C_{A}$ and $C_{B}$ exists, denoted $\phi_{I}\left(C_{A}, C_{B}\right)$, if $\phi_{D}\left(C_{A}, C_{S}\right) \wedge \phi_{D}\left(C_{B}, C_{S}\right)$ holds for some $C_{S}$.

Example 2. In the example Fig. 1, the concepts Supermarket and $O p$ are directly connected: Supermarket $\rightarrow_{M_{C}}$ Shop $\rightarrow_{M_{P}} \exists$ hasOp $\rightarrow_{M_{R}}$ Op. On the other hand, GuestGarden and Wlan are indirectly connected: GuestGarden $\rightarrow_{M_{C}}$ QVal $\rightarrow_{M_{P}}$ $\exists$ hasQVal $\rightarrow_{M_{R}}$ SpatialFeat $\leftarrow M_{R} \exists$ has $Q V a l \leftarrow M_{P} Q V a l \leftarrow M_{C}$ Wlan.

In general, several sequences that witness $\phi_{D}\left(C_{A}, C_{B}\right)$ resp. $\phi_{I}\left(C_{A}, C_{B}\right)$ exist.

Automatic Completion, Detection, and Combination of Keywords. As we get a sequence of entered strings $E=\left(e_{1}, e_{2}, \ldots, e_{n}\right)$, we need several steps to create the completed keywords, as the strings could be prefixes or misspelled.

First, we obtain the set of labels $L \subseteq \mathcal{N}$ by applying the prefix function $f_{P}\left(e_{i}\right)$ for every $e_{i} \in E$. Second, we build several levels of labels $L_{1}, \cdots, L_{m}$ based on the size of the subsets of $L$. As every $L_{i}$ has the subsets $L_{i, 1}, \cdots, L_{i, o}$ of the same size, we check for every $L_{i, j}$, if every pair of concepts (assigned to the labels of $L_{i, j}$ ) is syntactically connected at least in one direction. If we have found a $L_{i, j}$ with connected concepts, we add all sets of $L_{i}$ (which are connectable) to the results. This is done by concatenating the labels of every set of $L_{i}$ and add them to the result strings. 
By introducing an iterative algorithm, we return the largest possible combinations of keywords, thus excluding misspelled strings. However, we have in the worst-case exponentially many connectivity checks in the lengths of $E$.

Example 3. Given $E=$ (rest, in, non-smok), we obtain the labels $L=$ $\{$ restaurant, indian food, intl food, non-smoking\}. The first level of $L$ contains the sets $L_{1,1}=$ \{restaurant, indian food, non-smoking $\}$ and $L_{1,2}=$ $\{$ restaurant, intl food, non-smoking $\}$. The concepts assigned to them are $C_{1,1}=$ $\{$ Restaurant, IndianCuisine, NonSmoking $\}$ and $C_{1,2}=\{$ Restaurant, IntlCuisine, NonSmoking $\}$. Then, we check for $C_{1,1}$, if every pair $\left(C, C^{\prime}\right), C \neq C^{\prime} \in C_{1,1}$, is syntactically connected, and likewise for $C_{1,2}$. The first two pairs are directly connected and the last pair is indirectly connected by the common subsumer SpatialFeat. Hence, the concepts in $C_{1,1}$ (and in $C_{1,2}$ ) are connectable. Then, we concatenate $L_{1,1}$ (resp. $L_{1,2}$ ) and add the strings to the results.

\section{Refinement of Conjunctive Query Generation}

While FO-rewritability of CQ answering over DL-Lite ${ }_{R}$ KBs implies tractable data complexity, the size of the rewriting can increase exponentially with the number of atoms in the input CQ. Empirical findings [20] are that queries with more than 5-7 atoms can lead to large UCQs (e.g., unions of thousands of CQs) which cannot be handled by current RDBMS. Similar problems emerge with our generated SCQ (Sec. 8). One reason is the completion step in the SCQ generation. The generated SCQ can be too general, as we complete the intermediate sequence $K^{\prime}$ (Sec. 5) with the concept SpatialFeat and role $h a s Q V a l$, which are at the top-level (by our meta-model) of an ontology.

The refinement $O_{Q}$ of the completion step is applied on every ontological subquery of a SCQ of the form $S\left(x_{1}\right) \wedge R_{1}\left(x_{1}, y_{1}\right) \wedge C_{1}\left(y_{1}\right) \wedge \ldots \wedge R_{n}\left(x_{n-1}, y_{n}\right) \wedge C_{n}\left(y_{n}\right)$, where $S \sqsubseteq$ SpatialFeat, $\left\{R_{1} \ldots, R_{n}\right\} \sqsubseteq$ hasQVal, and $\left\{C_{1}, \ldots, C_{n}\right\} \sqsubseteq$ QualAttribute holds. It is based on the following ideas:

- Reduce the concept and role hierarchies: every edge in a path of $\phi_{D}$ or $\phi_{I}$ is an inclusion assertion, which increases the size of the rewritten UCQ; in particular, role inclusions can cause an exponential blow up [7];

- keep connectivity: by choosing paths according to $\phi_{I}$, we keep the domain, range, mandatory participation, regarding the roles connecting $S$ to $\left\{C_{1}, \ldots, C_{n}\right\}$.

Before applying $O_{Q}$, note that so far, $S$ is a most common subsumer different from the top concept with respect to $\phi_{I}$; i.e., for every pairs $\left(S, C_{1}\right), \ldots,\left(S, C_{i}\right), \phi_{I}\left(S, C_{j}\right)$ holds for all $j$ and the sum of path lengths for $\phi_{I}\left(S, C_{j}\right)$ is maximal. Thus, we try to minimize the path lengths under the constraint that $\phi_{I}$ is fulfilled for all pairs $\phi_{I}\left(S, C_{j}\right)$.

Briefly, it works as follows. We start the refinement $O_{Q}$ by taking every subconcept $S_{i}$ of $S$. We choose a shortest path, say $p_{j}$, according to $\phi_{I}$ for every pair $\left(S_{i}, C_{j}\right)$, $1 \leq j \leq n$, and we add up all path lengths $\left|p_{j}\right|$ to $l e n_{S_{i}}$. Finally, we choose the $S_{i}$ with the lowest $l e n_{S_{i}}$ as a replacement of $S$ and $R_{1} \ldots, R_{n}$, where the latter are replaced with the roles appearing on the shortest paths $p_{j}$ for $S_{i}$.

Example 4. Let $q\left(x_{1}\right)$ be SpatialFeat $\left(x_{1}\right) \wedge$ has $Q \operatorname{Val}\left(x_{1}, y_{1}\right) \wedge \operatorname{ItalianCuisine}\left(y_{1}\right) \wedge$ has $Q \operatorname{Val}\left(x_{1}, y_{2}\right) \wedge$ NonSmoking $\left(y_{2}\right)$. For the pairs (Rest, ItalianCuisine) and (Rest, 
NonSmoking), we have a path $p_{1}$ of length 2 (Rest $\rightarrow \exists$ hasCuisine $\rightarrow$ ItalianCuisine) and another path $p_{2}$ of length 2 (Rest $\rightarrow \exists$ provides $\rightarrow$ NonSmoking). Hence, the refinement $O_{Q}$ produces the optimized query $q^{\prime}\left(x_{1}\right)$, as the original paths are both of length 3 and Rest is a subconcept of SpatialFeat: Rest $\left(x_{1}\right) \wedge \operatorname{hasCusine}\left(x_{1}, y_{1}\right) \wedge$ ItalianCuisine $\left(y_{1}\right) \wedge$ provides $\left(x_{1}, y_{2}\right) \wedge \operatorname{NonSmoking}\left(y_{2}\right)$.

We point out that after applying $O_{Q}$, we may lose completeness with respect to the original SCQ, as shown by the following example. Given a spatio-thematic KB containing ABox assertions Rest $\left(i_{1}\right)$, hasCuisine $\left(i_{1}, i_{2}\right)$, ItalianCuisine $\left(i_{2}\right)$, SpatialFeat $\left(i_{3}\right)$, has $Q \operatorname{Val}\left(i_{3}, i_{2}\right)$, and ItalianCuisine $\left(i_{2}\right)$, such that hasCuisine has defined domain Rest and range Cuisine. The query $q\left(x_{1}\right)=\operatorname{SpatialFeat}\left(x_{1}\right) \wedge \operatorname{has} Q \operatorname{Val}\left(x_{1}, y_{1}\right) \wedge$ ItalianCuisine $\left(y_{1}\right)$ evaluates to $\left\{i_{1}, i_{3}\right\}$. If we refine $q\left(x_{1}\right)$ to the SCQ $q^{\prime}\left(x_{1}\right)=$ $\operatorname{Rest}\left(x_{1}\right) \wedge$ hasCuisine $\left(x_{1}, y_{1}\right) \wedge$ ItalianCuisine $\left(y_{1}\right)$, we just get $\left\{i_{1}\right\}$ as a result. Informally, completeness can be lost if the ABox assertions are very general. One way to keep completeness is thus to impose conditions on the ABox, which ensure that ABox assertions have to fulfill certain conditions.

\section{Implementation and Experimental Results}

We have implemented a prototype of our keyword-based query answering approach. It is developed in Java 1.6 and uses PostGIS 1.5.1 (for PostgreSQL 9.0) as spatialextended RDBMS. For the FO-rewriting of DL-Lite ${ }_{R}$, we adapted OWLGRES 0.1 [22] to obtain the perfect rewriting (with PerfectRef) of a CQ and the TBox. We evaluate spatial atoms in two different ways (Sec.4), namely as $O_{D}$ by using the query evaluation of PostGIS or as $O_{I}$ as a built-in component of our query evaluation algorithm. For $O_{D}$, we use the PostGIS functions for evaluation, e.g., ST_Contains(x, y), and for $O_{I}$, we apply the functions of the JTS Topology Suite (http://tsusiatsoftware.net/jts).

As part of a consortium with AIT Mobility Department (routing), Fluidtime (UI), ITS Vienna Region (data and routing), we have integrated our prototype for the keywordbased query answering in the MyITS system for intention-oriented route planning (http://myits.at/). Currently, the following services are available:

1. Neighborhood routing, where a user desires to explore the neighborhood for a keyword-based query; and

2. Via routing, where a route is calculated between a given origin-destination pair via some POI, which is dynamically determined by a keyword-based query.

Scenario. Our benchmarks are based on the usage scenarios of MyITS, which has a DL-Lite ${ }_{R}$ geo-ontology with the following metrics: 324 concepts (with 327 inclusion assertions); 30 roles (with 19 inclusion assertions); 2 inverse roles; 23 (resp. 25) domains (resp. ranges) of roles; 124 normal individuals; a maximal depth of 7 (4) in the concept (role) hierarchy (http://www.kr.tuwien.ac.at/staff/patrik/ GeoConceptsMyITS-V0.9-Lite.owl). For the spatial objects, we added and mapped the POIs of greater Vienna contained in OSM $(\approx 70 \mathrm{k}$ instances $)$, in the Falter database $(\approx 3700$ instances $)$, and parts of the OGD Vienna data $(\approx 7200$ instances $)$. The annotation step created $\approx 18700$ individuals, which lead to $\approx 18700$ concepts and $\approx 26000$ role assertions. The low annotation rate of $23 \%$ is related to the exclusion of some OSM POIs (e.g., benches, etc.) and the ongoing extensions of the mapping framework. 
Table 2. Benchmark Results (Evaluation time in secs), unrefined results in parentheses

(a) Benchmark $B_{1}$

\begin{tabular}{rrrr}
\hline & Instances & Query Size & Time \\
\hline$Q_{1}$ & $106(109)$ & $438(2256)$ & $1.66(4.96)$ \\
$Q_{2}$ & $1623(1623)$ & $51(2256)$ & $1.23(5.59)$ \\
$Q_{3}$ & $204\left(-^{s}\right)$ & $28(71712)$ & $1.14\left(-^{s}\right)$ \\
$Q_{4}$ & $32\left(-^{m}\right)$ & $56\left(-^{m}\right)$ & $1.48\left(-^{m}\right)$ \\
$Q_{5}$ & $3\left(-^{m}\right)$ & $112\left(-^{m}\right)$ & $4.11\left(-^{m}\right)$ \\
\hline
\end{tabular}

(b) Benchmark $B_{2}$, time only with $O_{Q}$

\begin{tabular}{rrrrr}
\hline & Instances & Query Size & \multicolumn{2}{c}{ Time } \\
& & & $O_{I}$ & $O_{D}$ \\
\hline$Q_{6}$ & $93(93)$ & $2(2)$ & 1.54 & 19.3 \\
$Q_{7}$ & $378(378)$ & $4(4)$ & 2.22 & $-^{t}$ \\
$Q_{8}$ & $26\left(-^{s}\right)$ & $30(71714)$ & 3.37 & $-^{t}$ \\
$Q_{9}$ & $151(151)$ & $2(2)$ & 2.02 & $-^{t}$ \\
\hline
\end{tabular}

Experiments. We conducted our experiments on a Mac OS X 10.6.8 system with an Intel Core i7 2.66GHz and 4 GB of RAM. We increased shared_buffers and work_mem of PostgreSQL 9.0 to utilize available RAM. For each benchmark, the average of five runs for the query rewriting and evaluation time was calculated, having a timeout of 10 minutes, and a memout of $750 \mathrm{MB}$ for each run. The results shown in Table 2 present runtime in seconds and query size (number of atoms in the CQ), and use $-{ }^{s}$ to denote DB errors (e.g., the stack depth limit of Postgres 9.0 is reached), ${ }^{m}$ for Java heap space limit has been reached $(750 \mathrm{MB})$, and $-{ }^{t}$ for timeout.

Benchmarks. We designed the first benchmark $B_{1}$ based on keywords to measure the refinement $O_{Q}$ on CQ without spatial predicates. The queries used in $B_{1}$ are

$Q_{1}$ : (spar) matches individuals run by "Spar";

$Q_{2}$ : (guest garden) returns the individuals with a guest garden;

$Q_{3}$ : (italian cuisine, guest garden) retrieves individuals that serve italian cuisine (including Pizzerias, etc.) and have a guest garden;

$Q_{4}$ : (italian cuisine, guest garden, wlan) gives individuals of $Q_{3}$ that in addition provide WLAN; and

$Q_{5}$ : (italian cuisine, guest garden, wlan, child friendly) returns individuals of $Q_{4}$ that in addition are child-friendly.

As described above, the keywords are completed to SCQ prior to evaluation as described.

The benchmark $B_{2}$ aims at comparing the database (denoted $O_{D}$ ) and internal evaluation of spatial predicates (denoted $O_{I}$ ) under the refinement $O_{Q}$. Its queries are

$Q_{6}$ : (playground, within, park) returns playgrounds in a park;

$Q_{7}$ : (supermarket, next to, pharmacy) matches supermarkets next to a pharmacy;

$Q_{8}$ : (italian cuisine, guest garden, next to, atm, next to, metro station) gives individuals with Italian food and a guest garden, whereby these individuals are next to an ATM and a metro station. The nesting of the query is as previously defined (((italian cuisine, guest garden), next to $), \ldots$, metro station $)$; and $Q_{9}$ : (playground, disjoint, park) retrieves playgrounds outside a park.

As the results in Table 2 show, the refinement $O_{Q}$ is essential for feasibility. Without it, Java exceeds heap space limitation during perfect rewriting in most cases, and SQL queries become too large for the RDBMS. The ontology of our scenario is big, yet captures only a domain for cities using OSM, OGD Vienna, and Falter. 
As ground truth we assume the unrefined query. We lost completeness only in $Q_{1}$; this is due to three objects, which were tagged in OSM as shops but not supermarkets. With respect to the benchmark queries, the OSM tagging and our (heuristic) mapping has a minor effect on the completeness. Further, the results for $Q_{2}$ to $Q_{5}$ reflect the fact that adding keywords extends the selectivity of the query (smaller results), but enlarges the UCQ considerably.

We were surprised by the large difference between internal and external evaluation of the spatial relations. We would have expected the external evaluation by the RDBMS is more efficient. Rewritten SQL queries have a three-leveled nesting, which consists of spatial joins $\left(\bowtie_{S}\right)$ on the first, unions $(\cup)$ on the second, and normal joins $(\bowtie)$ on third level. It seems that standard query evaluation and optimization (in Postgres 9.0) are overwhelmed by such complex structures.

\section{Related Work and Conclusion}

Regarding SCQ, the closest to our work is [18], where crisp results for the combination of FO-rewritability of DL-Lite combined with the $R C C$-family (which offers qualitative models of abstract regions in topological space) are provided. For more expressive DLs, Lutz et al. [17] introduced the notion of $\omega$-admissibility, which allows the combination of $\mathcal{A L C}$ and $R C C 8$ [19], for subsumption testing. In PelletSpatial [21], the authors implemented a hybrid combination of $\mathcal{S H O I N}$ and $R C C 8$. We follow a different approach in which the spatial regions are considered as point sets as in [14 15]. However, we focus on scalable query answering (without distance primitives) and the related implementation issues. In this way, we face similar challenges as recent Geo-SPARQL engines did (e.g., Strabon [16] and Parliament [3]). However, we have a stronger focus on ontology-based data access than on linked open-data (with an RDF data model).

Keyword-based search on the Semantic Web is a well-covered field of research. A necessarily incomplete list of relevant approaches is SemSearch [24], XXploreKnow [23], and QUICK [25] which are general purpose search engines. The KOIOS [4], DOROAM [9], and the system of [2] support (text-based) spatial queries using ontologies. Our approach differs from these systems regarding the expressivity of DL-Lite, with the addition of spatial querying; the use of a meta-model for suitable query generation; and a focus on gradual extendibility with new data sources.

In this paper, we presented an extension of DL-Lite ${ }_{R}$ with spatial objects using pointset topological relations for query answering. The extension preserves FO-rewritability, which allows us to evaluate a restricted class of conjunctive queries with spatial atoms over existing spatio-relational RDBMS. Second, we provided a technique for the generation of spatial conjunctive queries from a set of keywords. For this, we introduced a combination of a meta-model and completion rules to generate "meaningful" queries. Third, we implemented a prototype and performed experiments to evaluate the applicability in a real-world scenario. From our point of view, the first results are encouraging, as the evaluation time appeared to be moderate (always below 5 secs). Furthermore, our keyword-based approach is easy to extend, the text-based input is lightweight, and it has a reasonable precision through auto-completion and keyword combinations. However, precision could be improved by more advanced query expansion techniques (cf. [11]). 
Future research is naturally directed to variants and extensions of the presented ontology and query language. E.g., one could investigate how spatial conjunctive queries work over $\mathcal{E} \mathcal{L}[1]$ or Datalog $^{ \pm}$[5]. For our motivating application, the point set model was sufficient, but extending our approach with the DE-9IM model [10] would be appealing and introduce further spatial relations. Then, one could work on query expansion techniques and on refinement of query generation, in a way such that completeness is ensured. Finally, regarding the implementation, one could investigate the reason for the unexpected performance on very large queries with spatial functions and conduct further experiments on larger geospatial DBs, possibly comparing our approach to the mentioned Geo-SPARQL engines.

\section{References}

1. Baader, F., Brandt, S., Lutz, C.: Pushing the $\mathcal{E} \mathcal{L}$ envelope. In: IJCAI 2005, pp. 364-369. Morgan-Kaufmann Publishers (2005)

2. Baglioni, M., Masserotti, M.V., Renso, C., Spinsanti, L.: Improving geodatabase semantic querying exploiting ontologies. In: Claramunt, C., Levashkin, S., Bertolotto, M. (eds.) GeoS 2011. LNCS, vol. 6631, pp. 16-33. Springer, Heidelberg (2011)

3. Battle, R., Kolas, D.: Enabling the geospatial Semantic Web with Parliament and GeoSPARQL. Semantic Web Journal 3(4), 355-370 (2012)

4. Bicer, V., Tran, T., Abecker, A., Nedkov, R.: KOIOS: Utilizing semantic search for easyaccess and visualization of structured environmental data. In: Aroyo, L., Welty, C., Alani, H., Taylor, J., Bernstein, A., Kagal, L., Noy, N., Blomqvist, E. (eds.) ISWC 2011, Part II. LNCS, vol. 7032, pp. 1-16. Springer, Heidelberg (2011)

5. Calì, A., Gottlob, G., Pieris, A.: Towards more expressive ontology languages: The query answering problem. Artificial Intelligence 193, 87-128 (2012)

6. Calvanese, D., De Giacomo, G., Lembo, D., Lenzerini, M., Poggi, A., Rodriguez-Muro, M., Rosati, R.: Ontologies and databases: The DL-Lite approach. In: Tessaris, S., Franconi, E., Eiter, T., Gutierrez, C., Handschuh, S., Rousset, M.-C., Schmidt, R.A. (eds.) Reasoning Web 2009. LNCS, vol. 5689, pp. 255-356. Springer, Heidelberg (2009)

7. Calvanese, D., De Giacomo, G., Lembo, D., Lenzerini, M., Rosati, R.: Tractable reasoning and efficient query answering in description logics: The DL-Lite family. Journal of Automated Reasoning 39(3), 385-429 (2007)

8. Clementini, E., Sharma, J., Egenhofer, M.J.: Modelling topological spatial relations: Strategies for query processing. Computers \& Graphics 18(6), 815-822 (1994)

9. Codescu, M., Horsinka, G., Kutz, O., Mossakowski, T., Rau, R.: DO-ROAM: Activityoriented search and navigation with OpenStreetMaps. In: Claramunt, C., Levashkin, S., Bertolotto, M. (eds.) GeoS 2011. LNCS, vol. 6631, pp. 88-107. Springer, Heidelberg (2011)

10. Egenhofer, M.J., Franzosa, R.D.: Point set topological relations. International Journal of Geographical Information Systems 5(2), 161-174 (1991)

11. Fu, G., Jones, C.B., Abdelmoty, A.I.: Ontology-based spatial query expansion in information retrieval. In: Meersman, R., Tari, Z. (eds.) CoopIS/DOA/ODBASE 2005, Part II. LNCS, vol. 3761, pp. 1466-1482. Springer, Heidelberg (2005)

12. Gottlob, G., Leone, N., Scarcello, F.: The complexity of acyclic conjunctive queries. Journal of the ACM 48(3), 431-498 (2001)

13. Güting, R.H.: Geo-relational algebra: A model and query language for geometric database systems. In: Schmidt, J.W., Missikoff, M., Ceri, S. (eds.) EDBT 1988. LNCS, vol. 303, pp. 506-527. Springer, Heidelberg (1988) 
14. Haarslev, V., Lutz, C., Möller, R.: A description logic with concrete domains and a roleforming predicate operator. Journal of Logic and Computation 9(3), 351-384 (1999)

15. Kutz, O., Wolter, F., Zakharyaschev, M.: A note on concepts and distances. In: DL 2001. CEUR-WS, vol. 49 (2001)

16. Kyzirakos, K., Karpathiotakis, M., Koubarakis, M.: Strabon: A semantic geospatial DBMS. In: Cudré-Mauroux, P., et al. (eds.) ISWC 2012, Part I. LNCS, vol. 7649, pp. 295-311. Springer, Heidelberg (2012)

17. Lutz, C., Milicic, M.: A tableau algorithm for description logics with concrete domains and general TBoxes. Journal of Automated Reasoning 38(1-3), 227-259 (2007)

18. Özçep, Ö.L., Möller, R.: Scalable geo-thematic query answering. In: Cudré-Mauroux, P., et al. (eds.) ISWC 2012, Part I. LNCS, vol. 7649, pp. 658-673. Springer, Heidelberg (2012)

19. Randell, D.A., Cui, Z., Cohn, A.G.: A spatial logic based on regions and connection. In: KR 1992, pp. 165-176. Morgan Kaufmann (1992)

20. Rosati, R., Almatelli, A.: Improving query answering over DL-Lite ontologies. In: KR 2010, pp. 290-300. AAAI Press (2010)

21. Stocker, M., Sirin, E.: Pelletspatial: A hybrid RCC-8 and RDF/OWL reasoning and query engine. In: OWLED 2009. Springer, Heidelberg (2009)

22. Stocker, M., Smith, M.: Owlgres: A scalable OWL reasoner. In: OWLED 2008. Springer, Heidelberg (2008)

23. Tran, T., Cimiano, P., Rudolph, S., Studer, R.: Ontology-based interpretation of keywords for semantic search. In: Aberer, K., et al. (eds.) ISWC/ASWC 2007. LNCS, vol. 4825, pp. 523-536. Springer, Heidelberg (2007)

24. Uren, V.S., Lei, Y., Motta, E.: SemSearch: Refining semantic search. In: Bechhofer, S., Hauswirth, M., Hoffmann, J., Koubarakis, M. (eds.) ESWC 2008. LNCS, vol. 5021, pp. 874-878. Springer, Heidelberg (2008)

25. Zenz, G., Zhou, X., Minack, E., Siberski, W., Nejdl, W.: From keywords to semantic queries - incremental query construction on the semantic web. J. Web Semant. 7(3), 166-176 (2009) 